\title{
Design Methodology to Improve Human-Robot Coproduction in Small- and Medium-Sized Enterprises
}

\author{
Argun Cencen $^{(1)}$, Jouke C. Verlinden ${ }^{(0)}$, and J. M. P. Geraedts ${ }^{(1)}$
}

\begin{abstract}
In recent years, mass-customization and ondemand production have spread to larger ground. To accommodate these developments, manufacturing systems are being transformed to allow more flexibility and agility. One of the technologies that allow flexibility and agility is collaborative robots. The design and implementation of intelligent manufacturing systems (IMS) is a complex activity that requires bridging between disciplines. With the introduction of collaborative robots, new disciplines are added to this activity, which need to be linked to the existing design methods and procedures. Currently, the lack of these links is a bottleneck for small- and medium-sized enterprises that have limited resources for implementation. In this paper, we introduce a human-robot coproduction design methodology, with the aim of raising the capacity of designers for reasoning on collaboration between humans and robots in manufacturing. The methodology comprises four procedural steps: analysis, modeling, simulation, and evaluation, with specific methods, tools, and instruments. The methodology has been evaluated in a laboratory environment by performing a pilot study with designers. While the current implementation of the methodology and its instrumentation is limited, it has been shown that the methodology enables quick design iterations during the conceptual design phase of human-robot coproduction, thanks to procedures that have been tailored for this novel form of organizing and structuring production processes in IMS.
\end{abstract}

Index Terms-Collaborative robots, design methodology, human-robot collaboration (HRC), human-robot coproduction, intelligent manufacturing systems (IMS), small- and medium-sized enterprises.

\section{INTRODUCTION}

$\mathbf{I}$ T HAS become commonplace that product designs change with seasons, and today, it has become possible to

Manuscript received March 21, 2017; revised October 27, 2017 and March 27, 2018; accepted May 17, 2018. Date of publication May 22, 2018; date of current version June 12, 2018. Recommended by Technical Editor X. Chen. This work was supported by the European Community's Seventh Framework Programme (FP7/2007-2013) under Grant 609206. (Corresponding author: Argun Cencen.)

A. Cencen and J. M. P. Geraedts are with the Industrial Design Engineering Department, Delft University of Technology, Delft 2628 CD, The Netherlands (e-mail: a.cencen@tudelft.nl; j.m.p.geraedts@ tudelft.nl).

J. C. Verlinden is with the Faculty of Design Sciences, University of Antwerp, Antwerp 2000, Belgium (e-mail: jouke.verlinden@ uantwerpen.be).

Color versions of one or more of the figures in this paper are available online at http://ieeexplore.ieee.org.

Digital Object Identifier 10.1109/TMECH.2018.2839357 personalize/customize/produce products in an on-demand fashion. This holds true for personal as well as industrial products [1], [2]. The driving force behind this has been the achievements in intelligent manufacturing systems (IMS) [3]. However, while some manufacturing enterprises are able to transform their production processes to fit IMS, and realize mass-customization successfully, small- and medium-sized enterprise(s) (SME) in the manufacturing industry are facing challenges [4].

For an SME, production is typically short-cycled while the investments, infrastructure, and labor capacity impose a larger constraint compared to larger enterprises [5]. Recently collaborative robot (Cobot) technology has become economically more attractive and is being implemented by SME, in order to automate parts of production systems that were previously difficult to automate [6]-[9]. Regulations allow these robots to be implemented without the necessity for security fences that safeguard the humans that are required to work in the same environment as these robots [10]. However, in contrast to what the name suggests, these robots do not directly enable a human and a robot to work together in an effective and efficient manner. Achieving this requires a resource-intensive design activity [11], [12]. While these robots are relatively cheaper to purchase compared to their predecessors, and simple to configure/program, this bottleneck reduces their economic value, due to the amount of time, qualified engineers, and peripheral devices that are needed to integrate these robots in a production process and realize effective collaborative work throughout the production system [11].

In this paper, we introduce a human-robot coproduction design methodology to overcome the challenges faced in the SME context. In Section II, the notions of HRC design and humanrobot coproduction (HRC) are introduced, followed by a review of the literature that relates to these notions. In Section III the human-robot coproduction design methodology (HRCDM) is presented. In Section IV, the methodology is evaluated. Section V sets forth the conclusion of this paper and points to possible future improvements.

\section{BACKGROUND}

The future vision of IMS is that short production runs should be possible with the aim of batch sizes of one. This requires quick redesign and/or reconfiguration procedures. However, IMSs are complex and therefore their design process requires a multidisciplinary effort, and thereby, also a considerable amount 


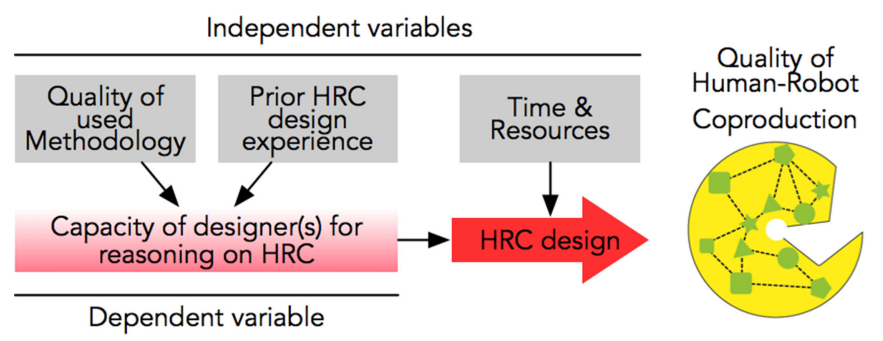

Fig. 1. Diagram showing the variables that influence HRC design. Yellow $=$ human, Green $=$ robot

of resources [13], [14]. With the introduction of Cobots, it has become more important to address the increased number of requirements related to collaborative work between machines/robots and operators. This type of work has previously been referred to as HRC [12].

Until now, the work has been going on to develop productive, flexible, intelligent, and quick-to-build production systems, with the ultimate goal of creating the lights-out-factory [15], [16]. However, co-operative production systems and humanmachine symbiosis have been highlighted as important areas for research and development [17]. In light of recent developments in robotics, and the introduction of Cobots, it has been noted that the physical and cognitive capabilities of humans such as dexterity, flexibility, adaptability, and problem solving are crucial skills in production systems, and therefore should become more explicit in the systems that are being developed [18], [19]. It has also been demonstrated that this form of work could benefit from social skills of humans [20]. However, in current practice, the task allocation between humans and robots in such systems is still cumbersome due to the lack of methods and tools to assess collaborative task performance from operational perspectives such as productivity, operator well-being, and training requirements [21].

Therefore, the goal of this work has been to develop a methodology that supports designers during the conceptual/structural design phase of production systems in the SME context, in which the implementation of Cobots is being considered.

The underpinning of this methodology is based on three assumptions, as illustrated in Fig. 1.

1) The capacity of designer(s) for reasoning on HRC is influenced by the quality of the used methodology and the prior HRC design experience.

2) An increase in reasoning capacity will be beneficial during HRC design, as time and resources are limited.

3) A more efficient and effective HRC design process will increase product quality and employability.

The HRC design methodology should consist of a design process, combined with methods and instruments that all are suitable for the SME context. Therefore, in this section, we review related work with respect to procedures, methods, and instruments that play a role in reasoning on HRC. Inspired by the successful integration of multiple domains of knowledge in the field of Mechatronics [22], the review spans multiple knowledge domains in order to cover the state-of-the-art relating to HRC. These domains include (but are not limited to) industrial and manufacturing engineering, systems and control engineering,

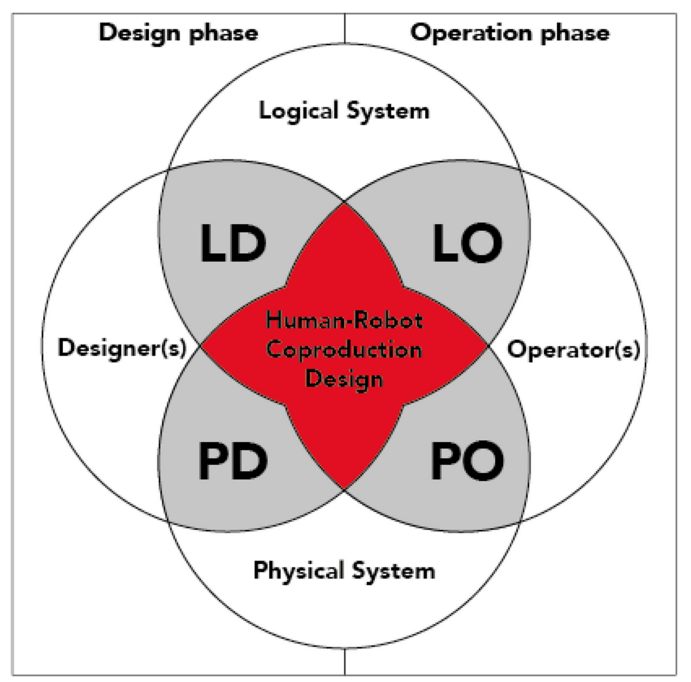

Fig. 2. Reasoning model for human-robot coproduction design.

mechatronics, human-computer interaction, ergonomics, and human factors.

As illustrated in the reasoning model for HRC design in Fig. 2, the state-of-the-art is presented in four categories: logical design (LD), physical design (PD), logical operation (LO), and physical operation (PO). The logical-physical design has been inspired by discussions in cyber-physical production systems (CPPS) literature [17], [23]. Next, a summary of the findings in each category is presented by which knowledge gaps are identified.

\section{A. Logical Design}

In the intersection between designer(s) and logical system, the developments related to abstract reasoning and criteria setting during HRC design were investigated.

For the HRC approach to be effective, when starting the design process, the product-related data need to be transformed into a format that can be interacted with throughout the design process. Several approaches have been proposed for creating an overview of assembly relations between parts of a product [24]. For example, Liaison graphs show physical interfaces between parts of a product [25]. This approach can be particularly helpful in determining the minimum and maximum amount of operations that the HRC system is should contain. The automatic extraction of Liaison information has been studied and several alternative solutions have been proposed such as, using motion planning and part interaction clusters, based on computer aided design (CAD) data [26]-[28]. To study the sequence of activities of assembling a product, the precedence diagram was introduced, in which the hierarchy between assembly steps can be seen [29]. With AND/OR graphs it was made possible to include additional attributes such as complexity of manipulation and stability of subassemblies [30]. Recently, the notion of assembly tiers was introduced in which an assembly can be split into groups of pars or subassemblies that can be assembled at the same time [31]. In this approach, fasteners of parts are also taken into consideration. 
Next to the data about the product, performance-related information is needed in order to guide the design process and to use in assessing generated design alternatives [21]. There have been numerous (experimental) attempts to provide SME with tools to perform calculations and make visualizations of return-oninvestment of HRC systems [32]-[34]. However, these do not go beyond basic mathematical calculations, and do not include details of the complexity that the design and integration process of HRC systems come paired with.

Concluding, there are already a number of methods for transforming product data into production-related data automatically. However, the formalization of data with respect to operators of HRC systems has received less attention. This reduces the capacity of reasoning of HRC designers.

\section{B. Physical Design}

In the intersection between designer(s) and physical system, the developments related to reasoning on the interactions that take place in the physical system (human-robot), and the learning capabilities of HRC systems were investigated.

Delta engineering is a new approach for contemplating the development of adaptations to existing systems [4]. It supports reuse of parts of the existing system, which decreases the development time. This is particularly valuable for SME, as their amount of resources and time is limited.

One of the requirements of implementing HRC in the context of SME is quick-setup and zero-configuration. In an ideal case, the system should work in the expected manner the first time it runs. This has been called plug-and-produce and plug-and-work [35], [36]. This method reduces the time that is further enhanced by systems that use Reinforcement Learning [6], [7], [37], [38]. This method reduces the amount of time that is needed for detailed and robust programming of systems during the design process. However, the implementation of learning systems is still a challenge to integrate in the context of SME [39].

The determination of the role of the human in a production workflow is a discussion that takes place during the design phase of the system [40]. So far, most Cobots that have been implemented in production settings have been mere weightcompensators [41]. However, this is changing with the introduction of more types of Cobots, including ones with multitasking skills. Therefore, the reconfiguration of systems based on an analysis of needed robot skills has only recently been introduced [42]. However, a similar approach for operator skills is missing. In most SME, an operator has multiple roles, covering multiple locations and machines. Therefore the role of an operator cannot be simplified/limited as an input/output during the design process of the system. Yet, often, operators are not taken explicitly as an element with multitasking capabilities in the design process of IMS. Currently, in most situations, designers focus on the "magic helper" role of humans. Therefore, HIMCoS-Human in the loop manufacturing control systems was introduced as a human-centered design approach [43]. Yet, this approach currently does not take the physical capabilities of operators into account.

In high-paced IMS, the cognitive and motoric capabilities of humans do not always fit well into the flow of the execution of all tasks in the system [43], [44]. This is also evident in the current categorization of human-robot collaborative configurations as, workplace sharing and workplace and time sharing systems [45]. This increases the importance of the mode of interaction and interfaces between operators and robots. Current research points toward teamwork as a plausible mode of interaction [46]. The information exchange between operators and IMS, and system awareness has also been studied in order to improve the interaction with operators [47]. Commercial hardware/software such as PASvisu allows web-based visualization of several layers of IMS at the same time and displays real-time and historical performance metrics related to the production system [48]. However such software requires active monitoring, and is currently not optimized for reducing cognitive load on operators.

Concluding, much work is being done on human-robot interaction and collaboration in the manufacturing context. However the specific requirements of SMEs related to multifunctionality and quick reconfigurability, result in an unbalance between the physical and cognitive load on operators, due to the limitations in usability of Cobots. Therefore, when designing HRC systems, it is essential to consider such limitations and to highlight the areas of increased physical and cognitive load for operators.

\section{Physical Operation}

In the intersection between physical system and operator, the developments related to the modeling and simulation of the HRC system, including its operators was investigated.

Product lifecycle management (PLM) is generally used to integrate multidisciplinary engineering activities related to product design and production. It is as a business-oriented, formally defined, holistic, digital, joined-up, and product-focused approach [49]. Yet, the consideration of the production system as a whole and the integration of this consideration into the product lifecycle management environment has only recently been taking shape [50].

In collaborative design, multiple representations and models are already used to act as boundary objects between stakeholders [51]. However, in current HRC design practice, this is not practiced [21].

Computer simulation is a powerful approach for analyzing and optimizing complex stochastic systems. In order to be able to perform computer simulations for new production requests, accurate models of the production system is mandatory. The creation of such models requires specialized personnel and building robust and realistic system models takes considerable amount of time.

In the context of IMS, simulations are used to simulate the operation of production processes, often for assessing robustness in case of production peaks [52]. While three-dimensional (3-D) simulations are common for the detailed design of IMS, the modeling and simulation of the interaction between various resources and servers of the system can be performed using discrete-event simulations (DES) [53]. Recently, detailed 3-D visualizations have become standard in DES [54], and the possibility to add visually realistic models of humans into system models has been made possible [55], [56]. In the future, for 
the successful integration of human models into these simulations, these visualizations should go beyond temporal and spatial domains. For example, audio can be added to these simulations as well [57]. One of the latest commercial PLM software suites in the context of manufacturing, "ABB Ability", integrates many aspects of IMS successfully [58]. However, similar to other software suites in this field, functionalities to model humans(operators) at cognition-level, and the capacity to support system designs for SME's, that are characterized by limited resources (time, budget, and operators) is still not being explicitly addressed.

Concluding, while abovementioned type of software has become very capable of addressing electromechanical and operational optimization problems, they neither provide methods nor functionalities to enable SME's in transitioning toward IMS and being part of Industry 4.0 [4].

\section{Logical Operation}

In the intersection between logical system and operator, the developments related to the performing evaluations and presenting the results of evaluations related to HRC were investigated.

Key performance indicator (KPI) is an established measure for assessing operational performance in many types of organizations, also in manufacturing SME [59]. The KPIs are still valuable measures in the era of product-service systems and aid in the evaluation of system designs that have complicated and intertwined requirements [60]. While generally being used to assess long-term goals, the balanced scorecard approach attempts to obtain a holistic judgment of operations in an organization and its application to manufacturing has been successful [61], [62]. While the scorecard covers aggregated and abstract indicators, in the context of HRC, another modality is necessary to convey the spatial and logical elements as well [63].

For the successful implementation of industrial HRC and effective teaming between operators and robots, operators need to be trained on the specific capabilities of the used robots, and empowered to handle degraded events, such as failures [64], [65]. Therefore, it is important to consider potential training costs and time during the evaluation of an HRC scenario.

If the HRC design process is considered as a closed-loop control system, the performance evaluation refers to the feedback that is sent to preceding controllers and systems. Yet, performance evaluation of logical operation of systems is perhaps one of the points that has received far less attention in comparison to methods for engineering and technical evaluation of systems.

Concluding, while an evaluation procedure at the end of a design process is almost trivial, in the case of HRC, this procedure should be grounded in methods used in the domain of human factors and should be addressed in developing an HRC design methodology as such.

\section{Human Robot Coproduction Design}

Having presented a gap in knowledge and a reasoning model for addressing this gap, we have reviewed the literature in the domains contributing to HRC design. Our review has shown that there are valuable methods and tools available for reasoning on aspects of HRC; however, that an overarching design approach is missing. We believe that, next to the technological developments, a systematic design methodology is essential for enabling SMEs to be part of IMSs. In this section, we present our HRC design process and present the HRCDM, which is our main contribution.

$\mathrm{Wu}$ divides production system design in four phases [66]: 1) analysis of situation, 2) setting of objectives, 3) conceptual modeling, and 4) evaluation of concepts. The proposed HRC design process consists of four interconnected subprocesses. In accordance with VDI 2221, it is extended with three inputs, one output, and one feedback loop [67]. In Fig. 3, our HRC design process is presented in the formalism of a control system. The main focus of this paper, HRCDM, is shown as an input that has influence on all subprocesses. In this section, HRCDM, as well as all inputs, processes, and outputs that are involved with the HRC design process will be elaborated and illustrated, with the aim of providing a reproducible process description that HRC designers can implement during their HRC design processes.

This section is structured as follows:First, the inputs of the design process are introduced. Next, the subprocesses are described, by discussing their own inputs,processes, and outputs. Finally, the output of the design process is described.

\section{A. Inputs of the HRC Design Process}

1) Input 1-Methodology: HRCDM has been developed to cover and support the four subprocesses that take place during HRC design. This methodology contains a collection of activities, methods, and instruments that can be used throughout these processes. Where, when, and which of these are used in the design process is elaborated on in the specific subprocess descriptions in the Section III-B-Processes.

Input 1 also contains information on the prior experience and know-how of designers in designing HRC systems, as well as their experience in using HRCDM. However, these factors have been left out of the scope of this study at this stage.

This input is used in analysis, modeling, simulation, and evaluation.

2) Input 2-Resource Constraints: In the future, as a result of flexibility and reconfigurability requirements, the production process will be product driven [18]. Therefore it is important to express the parameters that are used throughout the production process in terms of the product that needs to be made. Details on parts that are collected at this point should include(but is not limited to); size, form, weight, material, color, fragility, hard/soft, how do parts arrive (pallet, crate, bulk, and tray), level of varience between parts of the same family, and how parts/products should leave the production process. Besides this information, a table should be created that presents the assembly relationships between the parts of a product. The main goal is to determine how many operations will take place in the coproduction process. In order to create such table, methods mentioned in Section II-A-logical design should be used.

This input is used inanalysis.

3) Input 3-Performance Constraints: When an HRC design process is being performed, besides resource constraints, there are also constraints related to the performance of the HRC system that is targeted. In order to operationalize these constraints, 


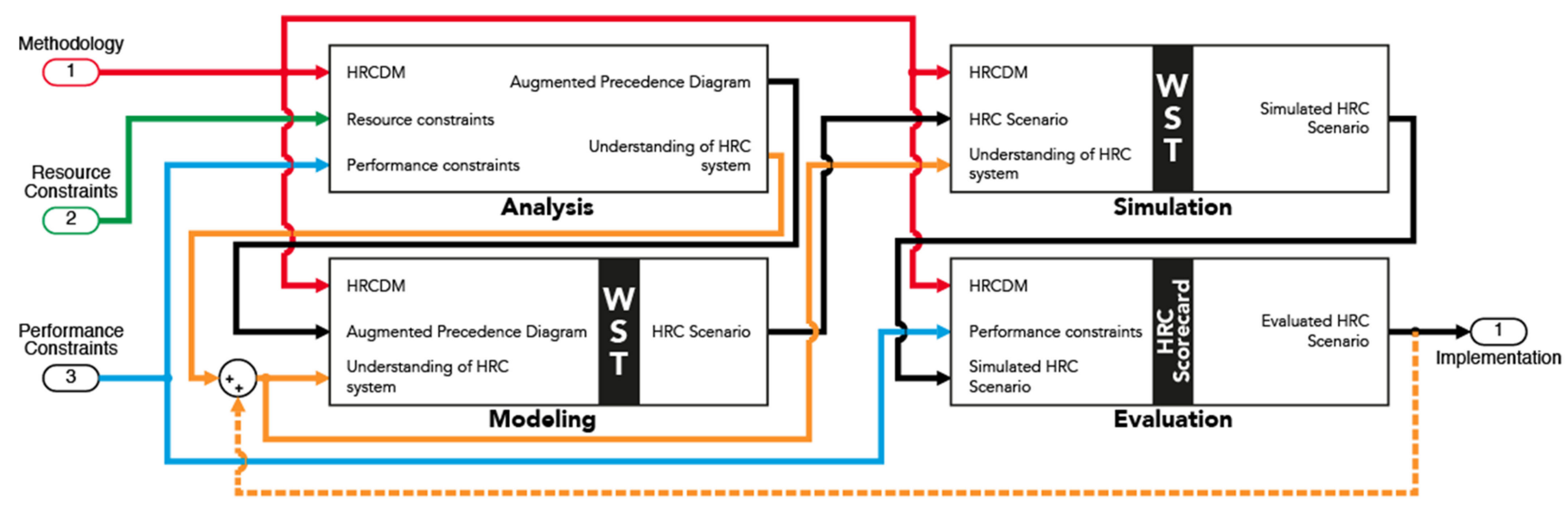

Fig. 3. Design process for HRC. Dashed line indicates a feedback.

it is practical to express these in the form of KPIs. KPIs are used throughout the HRC design procedure as a measure for reflection on the changes being made to the design of the system. We propose the categorization of HRC KPIs in three level/categories. Highest-level KPIs should include measures such as, amount of products to be produced per unit time and the cost of each product. On a lower level, the amount of operators in the system, the amount of times that the machines need to be serviced per unit time, should be included. On the lowest-level, operator well-being and ergonomic-related KPIs should be included.

This input is used in analysis, evaluation.

\section{B. Subprocesses of the HRC Design Process}

\section{1) Analysis:}

Inputs: The HRC design process starts with the analysis subprocess. In this process, the inputs resource constraints and performance constraints are processed using the support of HRCDM.

Processes: Depending on the initial state of the resource constraints, the designers should make a choice in the type of analysis methods to use (system existence $\mathrm{Y} / \mathrm{N}$ ). For an existing system, a thorough analysis of the existing resources such as machines and operators need to be performed. Also, current procedures need to be identified, such as workflows. Next, an analysis needs to be performed that addresses the constraints imposed by the new production scenario that is targeted. This scenario might concern a new product, or a better production performance. For a new system, a precedence analysis of the parts of the product should be performed (see Fig. 4).

At this point, the designers start considering the types of machines that can be used for production and the ideas and aspects related to the physical system start to emerge. This is also the moment that the "human" is introduced into the design process. During this activity, the roles of operators and the tasks of the robots in the system are allocated with the aim to eliminate any activities in the process that otherwise remain hidden, yet in real-time operation, claim cognitive attention and/or physical effort from operators.

Also, the amount of operators that should be in the control and production loops become apparent, and the physical and cog-

\begin{tabular}{|l|l|l|}
\hline \multicolumn{2}{|c|}{ Markers } \\
\hline part & Name & Has interface with \\
\hline A & Base & $B, C, D, E$ \\
\hline B & Pen1 & A \\
\hline C & Pen2 & A \\
\hline D & Pen3 & A \\
\hline E & Lid & A Steps \\
\hline \multicolumn{2}{|c|}{} \\
\hline Part(s) with least interfaces : BA, CA, DA, EA \\
\hline Which interface first? : BA=CA=DA \\
\hline \multicolumn{2}{|c|}{ Which other interface contain'BA"CA' or 'DA' : } \\
\multicolumn{3}{|c|}{ ABCDE } \\
\hline \multicolumn{2}{|c|}{ 4 Operations } \\
\hline
\end{tabular}

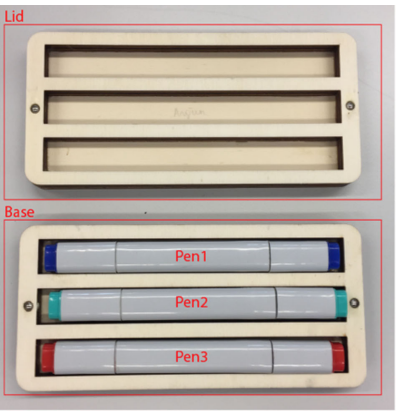

Fig. 4. Precedence analysis of a product consisting of five parts. The analysis gives insight into the interafece relationships between parts and aid in computing the amount and sequence of operations that will give shape to the production process.

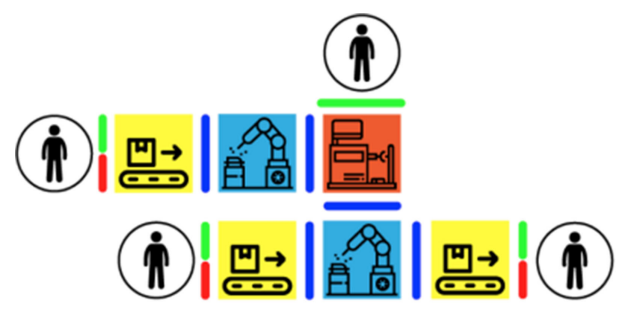

Fig. 5. Design criteria as captured by an augmented precedence diagram. In the diagram, the roles of the operators are made visible. Also, the types of interface between robots, machines, and operators are shown.

nitive interaction requirements between the robots and humans are addressed.

Another topic in this activity is the consideration of the types of tasks in the system.

During the Analysis process, the designers should predominantly make use of the methods mentioned in Section II-Alogical design.

Output: One of the outputs of the analysis process is augmented precedence diagram (APD) (see Fig. 5). Based on the production dependencies of the product's parts, tasks are defined. This APD depicts a network of the production tasks (material handling, automatic transformation, and collaboration). Connections are defined to specific human roles, with different interfaces between each task (cognitive/physical). Through this diagram, the designers can also consider which elements of the 


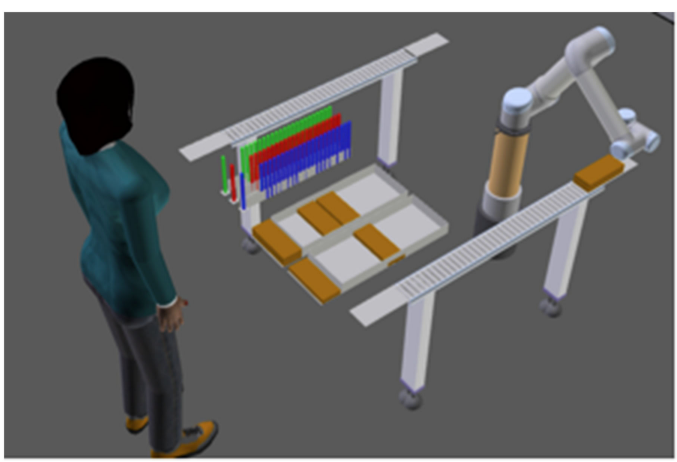

Fig. 6. Figure depicts a screen capture from the 3-D CAD software (visual components) that was used as one of the modules of WST. This module of the WST enables transitioning from a logical to physical model of the HRC system.

HRC system that should perform more robust during operation compared to others, due to limited modes of interaction with that element.

A second output of the analysis process is understanding of HRC system. While this output is not captured formally, it is transferred to the next process as tacit knowledge of the designer(s). In this process, the designers gain an initial understanding of the HRC system.

2) Modeling:

Inputs: In the modeling process, the inputs APD and understanding of HRC system are processed using the support of HRCDM.

Processes: In the modeling process, the activities are performed using the workflow simulation tool (WST), which is part of HRCDM (see Fig. 6). This tool aids in transforming and merging parameters of the physical and logical parts of the HRC system into a single digital model on which simulations can be performed. A pilot implementation of the tool has been iteratively developed and tested in close collaboration with automation integrators and was evaluated as being appropriate for the SME context [68]. The tool comprises of off-the-shelf software that enables modeling, simulation, and 3-D scanning within a handheld portable system [69]. The full-version of the tool encompasses the following functions.

1) An online repository containing automation templates, production machines, cobots, human models, etc.;

2) Facilitating the modeling of the entities captured in the APD using a visual programming language [70];

3) Concurrent development of 3-D CAD and discrete-event models of the HRC;

4) 3-D scanning of the environment and specific machinery;

5) Export of simulations in 3-D PDF format, to be shared with stakeholders.

During the modeling process, the designers can create alternative scenarios in which the production workflow is still valid, yet is performed through different distributions of roles between the operators and robots. Also in this process, the topic of endeffectors for Cobots, such as vacuum/mechanical grippers to be used can be addressed. The designers can choose suitable end-effectors from templates that are available in a component library, which is part of the WST, and configure these devices according to the specifications of the products that are being handled.

During the Modeling process, designers should predominantly make use of the methods mentioned in Section II-Bphysical design.

Output: The modeling process is aimed at generating an HRC scenario. An HRC scenario encompasses a detailed model of the HRC system, including logical attributes, such as sequences of activities, but also physical attributes, such as sized and place of elements of the system.

3) Simulation:

Input: In the simulation process, the inputs HRC scenario and understanding of HRC system are processed using the support of $H R C D M$.

Processes: In the simulation process, in coherence with the modeling process, the activities are performed using the workflow simulation tool (WST). WST is used in order to iteratively simulate and optimize the HRC scenario that was created in the modeling process. During simulation, the performance of the HRC scenario is simulated against time and by using the resource constraints as boundary conditions. This has been achieved in the WST by combining 3-D CAD simulation with discrete-event simulation. The 3-D CAD simulation allows the simulation of physical attributes of HRC, such as the reaching envelopes of robots and operators, and make use of actual programmable-logic controller programs that are installed in the system. The discrete-event simulation allows the simulation of HRC along a given time period and tracks the use of resources and the effects of extended durations of work on operators of the system based on models of actual operators based on historical statistical data that are available on the performance characteristics of that individual.

Some of these parameters can be expressed as upper and lower limits in an objective function. This enables the optimization of desired attributes of the HRC system. The WST is capable of suggesting optimizations to certain parameters of the HRC scenario, by running automated optimization calculations [71].

During the simulation process, the designers should predominantly make use of the methods mentioned in Section II-Cphysical operation.

Output: The output of the simulation process is the HRC scenario, with additional data on its performance in certain boundary conditions.

\section{4) Evaluation:}

Input: In the evaluation process, the inputs simulated $H R C$ scenario and performance constraints are processed using the support of HRCDM.

Processes: The evaluation process is performed using $H R C$ scorecards (see Fig. 7). HRC scorecards are instruments that support summarizing multiple qualities of HRC scenarios and present information in a clear and comprehensible manner. When multiple HRC scenarios are created and simulated, by viewing the scorecard of each scenario side-by-side, comparisons can be made between scenarios.

During the simulation process, designers should predominantly make use of the methods mentioned in Section II-Dlogical operation. 


\begin{tabular}{|l|c|c|c|}
\hline \multicolumn{5}{|c|}{ Scenario 1a } \\
\hline Operators & Investment & First product on: & Last product on: \\
\hline 2 & 10000 EUR & $23 / 03 / 18$ & $30 / 03 / 18$ \\
\hline \multicolumn{4}{|c|}{ Production } \\
\hline Products Per D. & $1000-2000$ & \\
\hline Cost per unit & $10-12$ EUR & \\
\hline Floor space & $75 \mathrm{~m} 2$ & Operators \\
\hline \multicolumn{4}{|c|}{ O) } \\
\hline Physical work & Cognitive work & Skilled work & Training \\
\hline HIGH & HIGH & LOW & LOW \\
\hline \multicolumn{4}{|c|}{ Suggested operators : } \\
\hline \multicolumn{4}{|c|}{ ID12,ID14,ID21,ID23 } \\
\hline
\end{tabular}

Fig. 7. Example HRC scorecard. The scorecard is divided into sections in order to provide a clear overview of the groups of scores. Also, some visual cues such as colors are used in order to indicate if a particular score is exceeding/below a critical limit.

Output: The output of the evaluation process is an evaluated HRC scenario. More details on this output are discussed in the next section.

\section{Output of the HRC design process}

When all subprocesses of the HRC design process has been performed, an evaluated HRC scenario is generated. This output consist of details of the logical and physical system such as, actual identificaiton of operators that will be functioning in the system, and the actual equipment that will be part of the physical system, and their availability. On top of the evaluated HRC scenario, this output includes bill of materials, in which information on all fixed and consumable elements of the system are presented. Where applicable and necessary, a customized parts list and the respective digital models of these parts are generated, such as 3-D printed grippers. A deployment manual contains information on the chronological order of steps to be taken during implementation. Also, an overview of the tasks that need to be taught to the robots by the operators and the physical/cognitive nature of these tasks is generated. Finally, a training schedule and related documents are generated for the operators.

Using this output, the choice to implement the system or to continue with design can be made.

\section{EVALUATION}

A pilot study was performed in a laboratory setting. Our hypothesis was that HRC design is influenced by the design methodology as presented in Fig. 1. This hypothesis is tested in an exploratory way, with a special focus on the developed output formats APD, HRC scenario, and HRC scorecards. During the pilot study, as an addition to Fig. 1, a "design brief" is used as a control variable.

\section{A. Method}

Three groups of four industrial design engineering students were participants of the pilot study. They were given a design brief, guided by the first author. As a case study, we could specify the following characteristics.

Design brief: In order to be able to generate the inputs resource constraints and performance constraints, the participants were asked to design their own products according to the following criteria.
1) The product should fit in the definition of a consumer product.

2) The product needs to fit in the SME context.

3) The products needs to fit in a predefined container in the final step of production.

4) The product needs to contain only several parts. The three products that resulted were as follows:

(i) A laser engraveable box with a custom selection of colored marker pens,

(ii) A set of candy boxes with a custom selection of candies.

(iii) Three boxes of a custom selection of alphabetic letters made out of chocolate.

The participants were asked to prepare a prototype of their product to be used in the final part of the exercise, in which they were asked to implement the HRC scenario that they have designed during the pilot study.

Methodology: The participants of the study followed the processes of HRCDM as described in Fig. 3 and finalized the pilot study by building an implementation of the HRC scenario that they designed. During the course of the exercise, an HRCDMexpert guided the teams.

Experience: The participants did not have any prior knowledge of the methodology or its constituents. The participants did not have any prior experience in designing HRC systems.

Time and Resources: The pilot study consisted of eight contact sessions with the groups. During each session, each group received $1 \mathrm{~h}$ of one-on-one guidance and feedback on the respective part of the HRC design process that the group was working on. Each team dedicated a total time of $40 \mathrm{~h}$ in the pilot study.

\section{B. Results}

In this section, details are given on the results of each specific HRC design process during the pilot study.

1) Prepilot Study Activities: Prior to the pilot study, after the design of products, the participants were also requested to design and build the gripper that will be attached to the Cobot that will handle the products as part of one of the tasks in the HRC scenario. The gripper needed to be produced using 3-D printing and it required the use of off-the-shelf suctions cups. The participants printed and assembled their 3-D printed grippers. These were tested with the subject product and optimized. This step was necessary in order to ensure a successful implementation phase.

2) Analysis: As the first step of the HRC design process, the participants created lists in which they summarized the required assembly steps for their products. Next, participants created APDs of their products. The APDs allowed the participants to visualize the amount and variety of steps that are involved in the production of the product they proposed. The APD allowed them to iteratively extend their analysis.

3) Modeling: In the modeling process, by reasoning on the APDs, the participants developed several (2) and (3) HRC scenarios. In order to specify the duration of activities related to specific tasks in the scenarios, estimations were made based on executing the task manually. By adding these durations in the created coproduction scenarios, adjustments were made to 


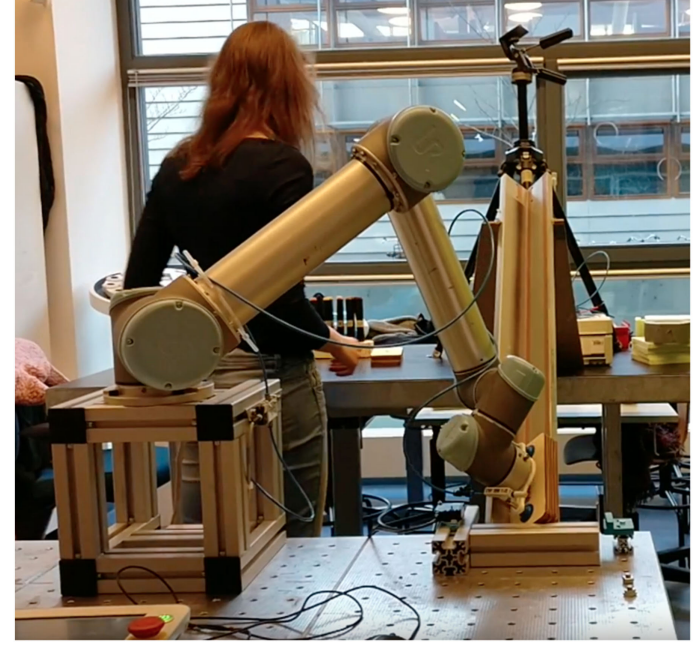

Fig. 8. Scene from the implemented HRC scenario of one of the students groups that took part in the pilot study, showing the collaborative robot, the 3-D printed gripper, the product, and the operator.

the sequence of events. The APD allowed the participants to elaborate on the changes being made to the design and the relative advantages and disadvantages of each design being created. Templates of best practices and components for the physical system were investigated and used for building HRC scenarios.

4) Simulation: In the simulation process, initially, the participants simulated their coproduction scenarios using the WST. This allowed the participants to observe the differences in dynamics of each HRC scenario that they proposed. Also, the layout of the workstation and the duration of tasks became more explicit with respect to the previous phase. As a final step, the HRC scenarios were optimized according to the space requirements and the available equipment for the final implementation.

5) Evaluation: In the evaluation process, the simulated scenarios were compared with each other by discussing the topics in the HRC scorecards verbally within the groups.

\section{Discussion}

As the final step of the exercise, the participants configured a robot cell to execute one of the robot tasks in the HRC scenario that was designed by the group (see Fig. 8). All three HRC systems performed one cycle (until one input buffer was depleted) of the coproduction scenario that the groups designed.

The time and resource restrictions during the pilot study played a crucial part in this evaluation. Only the part of the HRC system that would be implemented in the final phase was modeled. This resulted in an incomplete estimation of the real HRC scenario. In a follow-up study, the complete HRC system should be covered.

Also due to time restrictions, the subprocesses of HRCDM were not performed through extensive use of the methods described and alternating between methods. In this evaluation, the focus was on the newly developed output formats APD, HRC scenario, and HRC scorecards. Also, the evaluation of the WST was a priority.
The HRCDM expert assessed the appropriateness of the products according to the design criteria and the products were regarded suitable as input for the exercise of designing an HRC system for the SME. These products were solely created for the purpose of this exercise and therefore were not restricted by some resource and performance constraints that apply to reallife products/production, such as lead-times and costs of parts. Yet, the criteria in the design brief such as the use of a predefined container in the last process step ensured that there were sufficient boundary conditions posed to designers.

The used 3-D printed gripper was not modeled and used in the simulations. In a follow-up study, the gripper could be implemented into the WST.

In the instructions given to the participants, the possibility to generate tool paths for the robot by the WST was mentioned. However, these were not used in the actual implementation. Instead, by directly instructing the tool paths to the Cobot, the team could shorten the time for programming while exploring different movement strategies.

While the HRC scorecards were verbally discussed during the evaluation process in the pilot study, actual HRC ecorecards as depicted in the methodology were not used for this discussion. However, the HRC scorecards were generated based on the products and the corresponding HRC scenarios a posteriori (see Fig. 7). These HRC scorecards were addressed during a separate session with an expert. The participant in this evaluation was a human resource advisor from a major outsourcing company in the Netherlands, with more than ten years of experience in the manufacturing industry. The HRC scenarios and the corresponding HRC scorecards were presented to the participant on a laptop computer. The participant expressed the added value of the inclusion of operator-related information such as training requirements and mental/physical strain on operators.

\section{CONCLUSION AND FUTURE WORK}

In our review of literature and previous publications, we have demonstrated the knowledge gap in bridging the knowledge domains that are represented in the HRC design process. Recently, numerous research projects have taken place that focused on supporting the development of HRC systems. While these project developed new data-models that enable efficient and effective communication between the entities in an IMS [72], explored the modes of operation, interaction, and instruction between Cobots and operators [73], and enabling quick deployment of Cobots [74], none of these projects aimed at improving the capacity of designers for addressing cognitive and ergonomic aspects of work of humans in HRC systems. Also, the specific qualities and requirements of SME have only been addressed in a relatively small number of cases, compared to mass-production and large-scale factories.

Our introduction of the hypothesis in Fig. 1 demonstrates the importance of the used methodology in HRC design and the reasoning model that we present in Fig. 2, consisting of LO, LD, PO, and PD can enable others to lay a sufficient knowledge base when reasoning on HRC. 
The developed methodology does not aim to provide an exclusive list of methods to use. However the aim is to connect existing methods, tools, processes, and instruments with the dedicated processes of the HRC design process, with particular attention to the needs of the SME.

One of the most important aspects for increasing the speed of HRC design for SME is the existence of (digital) models of the production system. This enables real-time assessment of production workflows, and reduces biased and ungrounded reasoning on potential alterations to the system. Therefore, the methodology is aimed at stimulating the concurrent creation of interlinked physical and logical models of production systems. With the support of the purpose-built output formats, the design process is streamlined and a common ground is created for HRC designers to build upon, helping to reduce the knowledge gap further.

Results of the pilot study and heuristic evaluation with experts show that the methodology provides a common ground for stakeholders during the design process and it enables them to reason on various characteristics of $\mathrm{HRC}$ at the same time.

Yet, it has been observed that there are two shortcomings that need addressing, first the novel tools and instruments of HRCDM and, second, the accessibility for SME.

\section{A. Novel Tools and Instruments of HRCDM}

APD: Going from a product design to a functional HRC system, in a matter of hours is a challenging task. The Analysis process enables designers to handle incoming information in a systematic way. Yet, the APD can be improved to accommodate more parameters and data about the product, the process, and the operators. This will improve the quality and robustness of generated HRC Scenarios.

WST: In the current implementation, a software integration of the individual software components of the WST has not been realized. The workflow in which APD is transformed into discreteevent and CAD models needs to be refined. This will increase the usability of the WST and further reduce the design time, and increase number of design iterations.

HRC Scorecards: HRC Scorecards could be improved by integrating metrics that are specifically developed for humanrobot interaction, such as those created by Steinfeld et. al [75]. Susilawati already extended the scorecard to eight categories for lean manufacturing [76]. These categories could help to adapt scorecards for the context of HRC.

\section{B. Accessibility for SME}

In its current form, HRCDM comprises of a patchwork of procedures, methods, tools, and instruments that enable designers to reason on HRC. However, as the concept of a "Digital Twin" shows, a common ground is necessary in order to finalize the design-production-operation loop of IMS in the IndustrialInternet-of-Things era [77]. Therefore, one of the goals is to implement HRCDM in a PLM-like software environment. Integration of HRCDM in PLM will enable the automation of some of the reconfiguration tasks and reduce development time even further. While current industrial software allows the integration of PLM and robot/automation CAD software successfully, it is often too extensive to be deployed rapidly. However, it should be ensured that such tools are within reach of and usable by SMEs, for example, through pay-per-use licensing schemes.

\section{REFERENCES}

[1] T. Wasley et al., "Enabling rapid production and mass customisation of electronics using digitally driven hybrid additive manufacturing techniques," in Proc. Electron. Compon. Tech. Conf., 2016, pp. 849-856.

[2] "247TailorSteel - Custom-cut sheet metal, tube and bent products." [Online]. Available: https://www.247tailorsteel.com/en. [Accessed: 15-Mar2018].

[3] E. Brynjolfsson and A. McAfee, The Second Machine Age: Work, Progress, and Prosperity in a Time of Brilliant Tech. New York, NY, USA: W. W. Norton \& Company, 2014.

[4] H. H. Kagermann and H. Wahlster, "Recommendations for implementing the strategic initiative INDUSTRIE 4.0,'Berlin, Germany: Forschungsunion 2013.

[5] B. Siciliano and O. Khatib, Springer Handbook of Robotics. Berlin, Germany: Springer-Verlag, 2016.

[6] M. Denisa, A. Gams, A. Ude, and T. Petric, "Learning compliant movement primitives through demonstration and statistical generalization," IEEE/ASME Trans. Mechatron., vol. 21, no. 5, pp. 2581-2594, Oct. 2016.

[7] K. Miura, A. Matsui, S. Katsura, and S. Member, "Synthesis of motionreproduction systems based on motion-copying system considering control stiffness," IEEE/ASME Trans. Mechatron., vol. 21, no. 2, pp. 10151023, 2016.

[8] Z. Wang et al., "Design of a novel compliant safe robot joint with multiple working states," IEEE/ASME Trans. Mechatron., vol. 21, no. 2, pp. 1193 $1198,2016$.

[9] B. Olofsson, J. Antonsson, H. G. Kortier, B. Bernhardsson, A. Robertsson, and R. Johansson, "Sensor fusion for robotic workspace state estimation," IEEE/ASME Trans. Mechatron., vol. 21, no. 5, pp. 2236-2248, 2016.

[10] "Robots and robotic devices-Collaborative robots," ISO/TS 15066:2016, 2016.

[11] "Factory-in-a-Day," 2014. [Online]. Available: www.factory-in-a-day.eu. [Accessed: 01-Mar-2016].

[12] A. Cencen, K. van Deurzen, J. C. Verlinden, and J. M. P. Geraedts, "Exploring human robot coproduction," in Proc. IEEE Emerging Tech. Factory Automat., 2014, pp. 1-4.

[13] R. Jardim-Goncalves, D. Romero, and A. Grilo, "Factories of the future: challenges and leading innovations in intelligent manufacturing," Int. J. Comput. Integrated Manuf., vol. 30, no. 1, pp. 4-14, 2017.

[14] M. Prösser et al., "A new approach towards systems integration within the mechatronic engineering design process of manufacturing systems," Int. J. Comput. Integrated Manuf., vol. 26, no. 8, pp. 806-815, Aug. 2013.

[15] T. B. Sheridan, "Human supervisory control," in Design of Work and Development of Personnel in Advanced Manufacturing. Hoboken, NJ, USA: Wiley, 1994, pp. 79-102.

[16] D. B. Brann, D. A. Thurman, and C. M. Mitchell, "Human interaction with lights-out automation: a field study," in Proc. 3rd Anпи. Symp. Human Interaction Complex Syst., 1996, pp. 276-283.

[17] L. Monostori et al., "Cyber-physical systems in manufacturing," CIRP Annals-Manuf. Tech., vol. 65, no. 2, pp. 621-641, 2016.

[18] H. A. ElMaraghy M. F. Zaeh et al., ,"The cognitive factory," in Changeable Reconfigurable Manuf. Syst. - Springer Series Adv. Manuf., H. A. ElMaraghy, Ed. London, U.K.: Springer, pp. 355-371, 2009.

[19] A. Bannat, J. Gast, T. Rehrl, W. Rösel, G. Rigoll, and F. Wallhoff, "A multimodal human-robot-interaction scenario: Working together with an industrial robot," Berlin, Germany: Springer-Verlag, 2009, pp. 303-311.

[20] B. Sadrfaridpour, H. Saeidi, and Y. Wang, "An integrated framework for human-robot collaborative assembly in hybrid manufacturing cells," in Proc. IEEE Int. Conf. Robot. Automat., 2016, pp. 462-467.

[21] A. Cencen, J. Verlinden, and J. Geraedts, "Qualifying the performance of Human Robot coproduction at a relabling station," in Proc. Tools Methods Concurrent Eng., 2016, pp. 1-10.

[22] W. Bolton, Mechatronics: A Multidisciplinary Approach, vol. 10. New York, NY, USA: Pearson Education, 2008

[23] F. Perez, E. Irisarri, D. Orive, M. Marcos, and E. Estevez, "A CPPS architecture approach for Industry 4.0," in Proc. IEEE 20th Conf. Emerging Tech. Factory Automat., 2015, pp. 1-4.

[24] R. D. D. (Robert D. Reid and N. R. Sanders, Operations Management Hoboken, N.J, USA: John Wiley, 2010. 
[25] M. Hudson, A. Smart, and M. Bourne, "Theory and practice in SME performance measurement systems," Int. J. Oper. Production Manage., vol. 21, no. 8, pp. 1096-1115, Aug. 2001.

[26] H. Y. Lai and C. T. Huang, "A systematic approach for automatic assembly sequence plan generation," Int. J. Adv. Manuf. Tech., vol. 24, no. 9-10, pp. 752-763, 2004.

[27] C. Morato, K. N. Kaipa, and S. K. Gupta, "Improving assembly precedence constraint generation by utilizing motion planning and part interaction clusters," CAD Comput. Aided Design, vol. 45, no. 11, pp. 1349-1364, 2013.

[28] M. V. A. Raju Bahubalendruni, and B. B. Biswal, "Computer aid for automatic liaisons extraction from cad based robotic assembly," in Proc. IEEE 8th Int. Conf. Intell. Syst. Control: Green Challenges Smart Solutions, 2014 , pp. 42-45.

[29] B. Fox and K. Kempf, "Opportunistic scheduling for robotic assembly," in Proc. Int. Conf. Robot. Automat., 1985, pp. 880-889.

[30] L. S. H. De Mello, and A. C. Sanderson, "and/or graph representation of assembly plans," IEEE Trans. Robot. Automat., vol. 6, no. 2, pp. 188-199, Apr. 1990.

[31] G. Pintzos, C. Triantafyllou, N. Papakostas, D. Mourtzis, and G. Chryssolouris, "Assembly precedence diagram generation through assembly tiers determination," Int. J. Comput. Integrated Manuf., vol. 3052, pp. 1-13, Mar. 2016.

[32] "Robot Investment Tool-Investing in Robotics," [Online]. Available: http://www.robotinvestment.eu/. [Accessed: 21-Mar-2018].

[33] "Robotic ROI Calculator.” [Online]. Available: https://www.robotics.org/ robotics-roi-calculator. [Accessed: 21-Mar-2018].

[34] "Factory in a day - QuickScan." [Online]. Available: http://www.factoryin-a-day.nl/quickscan. [Accessed: 21-Mar-2018].

[35] M. Onori, N. Lohse, J. Barata, and C. Hanisch, "The IDEAS project: Plug and produce at shop-floor level," Assembly Automat., vol. 32, no. 2, pp. 124-134, 2012.

[36] M. Schleipen, E. Selyansky, R. Henssen, and T. Bischoff, "Multi-level user and role concept for a secure plug-and-work based on OPC UA and AutomationML," in Proc. IEEE 20th Conf. Emerging Tech. Factory Automat., 2015, pp. 1-4.

[37] K. Arviv, H. Stern, and Y. Edan, "Collaborative reinforcement learning for a two-robot job transfer flow-shop scheduling problem," Int. J. Production Res., vol. 7543, pp. 1-14, Jan. 2015.

[38] M. Keshmiri and W. F. Xie, "Image-based visual servoing using an optimized trajectory planning technique," IEEE/ASME Trans. Mechatron., vol. 22, no. 1, pp. 359-370, Feb. 2017.

[39] C. Faller and D. Feldmúller, "Industry 4.0 learning factory for regional SMEs," Procedia CIRP, vol. 32, no. Clf, pp. 88-91, 2015

[40] M. G. Jr and M. P. Groover, Automation, Production Systems and Computer-Aided Manufacturing. Englewood Cliffs, NJ, USA: PrenticeHall, 1980.

[41] A. Cherubini, R. Passama, A. Crosnier, A. Lasnier, and P. Fraisse, "Collaborative manufacturing with physical human-robot interaction," Robot. Comput.-Integrated Manuf., vol. 40, pp. 1-13, 2016.

[42] M. R. Pedersen et al., "Robot skills for manufacturing: From concept to industrial deployment," Robot. Comput.-Integrated Manuf., vol. 37, pp. 282-291, 2016.

[43] P. Trentesaux, D. Millot, D. Trentesaux, and P. Millot, "A human-centred design to break the myth of the 'Magic Human' in intelligent manufacturing systems," Studies Comput. Intell., vol. 640, pp. 103-113, 2016.

[44] A. Cencen, J. Verlinden, and J. Geraedts, "Characterizing the state of the art of human-robot coproduction," in Social Robotics (Lecture Notes in Computer Science, vol. 9388), A. Tapus, E. André, J. C. Martin, F. Ferland, M. Ammi, Eds. Cham, Switzerland: Springer, 2015.

[45] J. Krüger, T. K. Lien, and A. Verl, "Cooperation of human and machines in assembly lines," CIRP Annals-Manuf. Tech., vol. 58, no. 2, pp. 628-646, 2009.

[46] V. Groom and C. Nass, "Can robots be teammates?: Benchmarks in human-robot teams," Interaction Studies, vol. 8, no. 3, pp. 483-500, 2007.

[47] C. Escande, T. Chettibi, R. Merzouki, V. Coelen, and P. M. Pathak, "Kinematic calibration of a multisection bionic manipulator," IEEE/ASME Trans. Mechatron., vol. 20, no. 2, pp. 663-674, 2015.

[48] "Pilz-PASVisu Visualisation software." [Online]. Available: https://www. pilz.com/en-US/products-solutions/automation-system-pss4000/visualisation. [Accessed: 15-Mar-2018].

[49] J. Stark, Product Lifecycle Management. Cham, Switzerland: SpringerVerlag, 2015

[50] F. Ríos, J. C., Hernández, M. Oliva, and F. Mas, "Product avatar as digital counterpart of a physical individual product: Literature review and im- plications in an aircraft," in Proc. 22nd ISPE Inc. Int. Conf. Concurrent Eng., 2015, pp. 657-666.

[51] F. Smulders, L. Lousberg, and K. Dorst, "Toward different communication in collaborative design," Int. J., vol. 1, pp. 352-367, 2008.

[52] A. Negahban and J. S. Smith, "Simulation for manufacturing system design and operation: Literature review and analysis," J. Manuf. Syst., vol. 33 , no. 2, pp. 241-261, 2014.

[53] D. Mourtzis, M. Doukas, and D. Bernidaki, "Simulation in manufacturing: Review and challenges," Procedia CIRP, vol. 25, no. C, pp. 213-229, 2014

[54] Y. Zhong and B. Shirinzadeh, "Virtual factory for manufacturing process visualization," in Proc. 7th Asia-Pacific Conf. Complex Syst., Complex Open Systems Research Network, Cairns, Queensland, Dec. 6-10, 2004 pp. 557-578

[55] P. Tsarouchi, S. Makris, and G. Chryssolouris, "Human-robot interaction review and challenges on task planning and programming," Int. J. Comput. Integrated Manuf., vol. 29, no. 8, pp. 916-931, 2016.

[56] P. (Pete) Dode, M. Greig, S. Zolfaghari, and W. P. Neumann, "Integrating human factors into discrete event simulation: A proactive approach to simultaneously design for system performance and employees' well being,' Int. J. Production Res., vol. 54, no. 10, pp. 3105-3117, 2016.

[57] X. Yang, R. Malak, C. Lauer, and C. Weidig, "Manufacturing system design with virtual factory tools," Int. J. Comput. Integrated Manuf., vol. 28 , no. 1 , pp. 25-40, 2015

[58] "ABB Ability Software Suite." [Online]. Available: http://new.abb. com/abb-ability. [Accessed: 15-Mar-2018].

[59] P. Garengo, S. Biazzo, and U. S. Bititci, "Performance measurement systems in SMEs: A review for a research agenda," Int. J. Manage. Rev., vol. 7, no. 1, pp. 25-47, Mar. 2005.

[60] D. Mourtzis, S. Fotia, and E. Vlachou, "PSS design evaluation via KPIs and lean design assistance supported by context sensitivity tools," Procedia CIRP, vol. 56, pp. 496-501, 2016.

[61] A. Butler, S. Letza, and B. Neale, "Linking the balanced scorecard to strategy," Long Range Planning, vol. 30, pp. 242-253, 1997.

[62] R. S. Kaplan and D. P. Norton, The Balanced Scorecard: Translating Strategy into Action. Cambridge, MA, USA: Harvard Univ. Press, 1996.

63] W. Kühn, "Digital factory - Simulation enhancing the product and production engineering process," in Proc. Winter Simulation Conf., 2006 pp. 1899-1906.

[64] G. Charalambous, "The development of a human factors tool for the successful implementation of industrial human-robot collaboration," Thesis, Cranfield Univ., 2014

[65] G. Charalambous, S. Fletcher, and P. Webb, "Development of a human factors roadmap for the successful implementation of industrial human-robot collaboration," in Proc. Advances Ergonom. Manuf.: Manag. Enterprise Future, 2016, pp. 195-206.

[66] B. Wu, Manufacturing systems design and analysis. Berlin, Germany, Springer-Verlag, 2012

[67] VDI, "VDI 2221 Methodik zum entwickeln und kosntruieren technischer systeme und produkte," Verein Deutscher Ingenieure. pp. 1-44, 2013.

[68] J. C. Dukalski et al., "Portable rapid visual workflow simulation tool for human robot coproduction," in Proc. Int. Conf. Flexible Autom. Intell. Manuf., 2017, vol. 11, no. June, pp. 185-197.

[69] "Visual components premium - The factory simulation solution." [Online]. Available: http://www.visualcomponents.com/visual-components4-0/premium/. [Accessed: 15-Mar-2018].

[70] S. Mitkin, "DRAKON-Erlang: Visual functional programming," [Online]. Available: http://www.drakonhub.com. [Accessed: 15-Mar-2018].

[71] S. Behbahani and C. W. De Silva, "Mechatronic design evolution using bond graphs and hybrid genetic algorithm with genetic programming," IEEE/ASME Trans. Mechatron., vol. 18, no. 1, pp. 190-199, Feb. 2013.

[72] "European commission: CORDIS: Projects and results: Holistic, extensible, scalable and standard virtual factory framework," [Online]. Available: https://cordis.europa.eu/project/rcn/97739_en.html. [Accessed: 27Mar-2018]

[73] "LIAA Project: Home." [Online]. Available: http://www.projectleanautomation.eu/. [Accessed: 23-Mar-2018].

[74] “SME Robotics." [Online]. Available: http://www.smerobotics.org. [Accessed: 01-Mar-2016].

[75] A. Steinfeld, T. Fong, and D. Kaber, "Common metrics for human-robot interaction," in Proc. 1st ACM SIGCHI/SIGART Conf. Human-Robot Interaction, 2006, no. 1, pp. 33-40.

[76] A. Susilawati, J. Tan, and D. Bell, "Develop a framework of performance measurement and improvement system for lean manufacturing activity," Int. J. Lean Thinking, vol. 4, no. 1, pp. 51-64, 2013. 
[77] Z. Bi, L. Da Xu, and C. Wang, "Internet of things for enterprise systems of modern manufacturing," IEEE Trans. Ind. Inform., vol. 10, no. 2, pp. 15371546, May 2014.

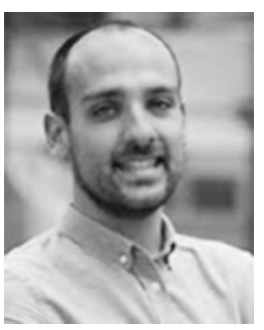

Argun Cencen, received the B.Sc. and M.Sc. degrees in industrial design engineering from Delft University of Technology, Delft, Netherlands, in 2010 and 2012, respectively. He is currently working toward the Ph.D. degree.

His research focuses on human-robot coproduction. The aim of his research is to support the design process of factories of the future.

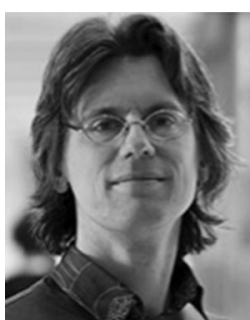

Jouke C. Verlinden received the M.Sc. degree in computer science in 1993 and has a background in interactive computer graphics and virtual reality.

His current work focuses on 'Augmented Matter in Context' to enable embodied dialogues by bridging physical and digital boundaries.

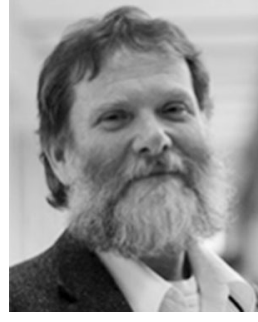

Jo M. P. Geraedts received the M.Sc. degree from Eindhoven University of Technology, Eindhoven, Netherlands, in 1976, and the Ph.D. degree from University of Nijmegen, Nijmegen, Netherlands, in 1983, both in physics.

From 1983 to 2017, he was with Océ-van der Grinten N.V.

Dr. Geraedts is the Chair of Mechatronics Design at TU Delft, where he leads research in 3-D scanning, 3-D printing, and human-robot interaction. 